\title{
Deutsch als Sprache der (Geistes-)Wissenschaften Ein neues Lehrbuch zur Didaktik der deutschen Wissenschaftssprache
}

\section{Katja Kernjak}

Since the winter semester 2010/2011 the students at the Palacký University can choose to study in a new program at the German department: German as the language of humanities. They can acquire a comprehensive knowledge of the basics of various humanities and thereby gain an overall foundation for philological research. Interdisciplinary academic work is required at the same time. The motivation leading to the creation of this study program stems from the awareness of tradition: German language and the German scientific tradition provided significant stimuli for Czech humanities.

An important part of the program is represented by special practical language seminars dealing with German academic language equipping students with necessary linguistic knowledge. A workbook serving this purpose was compiled by the members of the German department. The intention of this book is to draw attention to field specific terminology and to introduce language elements of scientific communication across a whole spectrum of disciplines. In addition to this it offers clues and instructions for academic writing.

German as the language of humanities - Czech humanities - German at Palacky University German scientific tradition - textbook Übungsbuch zur deutschen Wissenschaftssprache

An der Palacký-Universität können Studierende seit Wintersemester 2010/2011 im Rahmen des Germanistik-Studiums einen neuen Schwerpunkt wählen: Deutsch als Sprache der Geisteswissenschaften. Sie können dabei ein umfassendes Grundwissen aus verschiedenen geisteswissenschaftlichen Disziplinen und damit eine breite Grundlage für philologische Forschung erwerben. Gleichzeitig wird damit auch interdisziplinäres Arbeiten gefördert.

Die Motivation für die Ausarbeitung dieses Schwerpunktes geht nicht zuletzt aus dem Bewusstsein historischer Traditionen hervor: das Deutsche und die deutsche Wissenschaftstradition waren für die Geisteswissenschaften in Tschechien maßgeblicher Impulsgeber.

Wichtiger Bestandteil des Studienangebotes sind spezielle Sprachübungen zur Wissenschaftssprache Deutsch, die den Studierenden das nötige sprachliche Rüstzeug mitgeben sollen. Dafür wurde am Lehrstuhl ein Übungsbuch erstellt, das zum einen auf die Thematik der fachspezifischen Terminologien sensibilisieren soll, vor allem aber fächerübergreifende sprachliche Elementen der wissenschaftlichen Kommunikation behandelt. Darüber hinaus bietet es auch Hinweise und Handreichungen zur Gestaltung wissenschaftlicher Texte.

Deutsch als Sprache der Geisteswissenschaften - Geisteswissenschaften in Tschechien Deutsch an der Palacký-Universität - deutsche Wissenschaftstradition - Übungsbuch zur deutschen Wissenschaftssprache - Studienschwerpunkt Deutsch

\section{Einleitendes}

Am Institut für Germanistik können angehende Studierende seit dem Wintersemester 2010/2011 im Rahmen des Germanistikstudiums einen neuen 
Schwerpunkt wählen: Deutsch als Sprache der Geisteswissenschaften. Diese Vertiefung (oder besser gesagt: Erweiterung) soll den Studierenden über die klassischen Inhalte des Germanistikstudiums hinaus zusätzliche Kompetenzen auf zwei Ebenen bieten, nämlich auf einer ,inhaltlich'-geisteswissenschaftlichen und auf der sprachlichen Ebene. Im Folgenden möchte ich zunächst die Hintergründe schildern, die zur Konzeption des neuen Studienplans führten und eben diesen anschließend kurz skizzieren. Im Zentrum dieses Aufsatzes steht aber ein Lehr- bzw. Übungsbuch, das eigens für den Sprachunterricht bzw. die Sprachübungen im Rahmen des Schwerpunktes erarbeitet wurde.

\section{Die Hintergründe}

Die deutsche Sprache befindet sich in Tschechien in einer prekären Lage, die sich tendenziell noch weiter verschlechtern wird. Die Gründe dafür sind vielfältig: Zum einen sehen wir - wie auch in anderen Ländern - die Tendenz zum Englischen als erste (und damit wichtigste) Fremdsprache. Das macht sich auch in den Geisteswissenschaften bemerkbar, wo immer häufiger auf das Englische als ,Lingua franca' zurückgegriffen wird. Zum anderen muss aber auf historische Kontexte und Entwicklungen verwiesen werden, die bis in die Gegenwart wirken; das Deutsche hat auf dem Gebiet der heutigen Tschechischen Republik zwar eine lange, aber eben auch ambivalente Geschichte. Ein auch nur annähernd erschöpfender Überblick ist an dieser Stelle freilich nicht möglich, prägende Momente bzw. Aspekte sollen aber schlagwortartig genannt werden (im Bewusstsein der Unzulänglichkeit, die bei so stark verkürzten Aussagen unvermeidlich auftritt): Einerseits das Deutsche als ,Herrschaftssprache' in der Zeit der Habsburger Monarchie und das Deutsche als Sprache der nationalsozialistischen Okkupation zwischen 1939 und 1945. Andererseits aber auch damit einhergehende bzw. damit verbundene Abgrenzungsund Abwehrbewegungen auf tschechischsprachiger Seite, zu der traurige Höhepunkte wie die Vertreibung der deutschsprachigen Bevölkerung nach 1945 und das konsequente Totschweigen des deutschsprachigen Teiles der tschechischen Geschichte und Kultur in der Zeit des Kommunismus gehören. ${ }^{1}$

Durch diese Aspekte und ihre bis heute wirkenden Folgen gerät eines leicht in Vergessenheit oder rückt jedenfalls in den Hintergrund: die Deutsche Sprache und die Wissenschaftstradition der deutschsprachigen Länder bot sowohl für die Kultur als auch die Entwicklung der Geisteswissenschaften auf dem Gebiet der heutigen Tschechischen Republik eine wesentliche Anregung: „In den weitaus meisten Fächern der Geisteswissenschaften gingen in Böhmen die Impulse zu ihrer Gründung ehedem von Deutschland aus. Das Humboldtsche Wissenschaftsideal war auch hier

1 Einen guten Überblick über diese Problematik bzw. ihre Entwicklung bietet, fokussiert auf Österreich und Tschechien, Payrleitner (1990/2003). 
ein Leitparadigma durch das gesamte 19. Jahrhundert bis weit ins 20. Jahrhundert hinein". ${ }^{1}$ Tschechische Geisteswissenschaftler, die der deutschen Sprache nicht mächtig sind, stoßen unweigerlich früher oder später auf ein Problem: Ein nicht geringer Teil der Grundlage ihrer Forschung, also Quellen und Primärtexte, wurden in deutscher Sprache verfasst. Dies gilt insbesondere für Historiker, Philosophen und Literaturwissenschaftler. Das Deutsche ist in Tschechien mehr als nur eine ,Fremdsprache ${ }^{\prime}$ - es ist Teil der tschechischen Kultur und Wissenschaft, so paradox dies auf den ersten Blick auch scheint und so stark dies von nicht wenigen Menschen in Tschechien (auch heute noch) verdrängt wird.

Diese hier nur sehr oberflächlich berührten Überlegungen führten am Institut für Germanistik der Palacký-Universität in Olomouc/Olmütz (unter anderem) zu dem Entschluss, einen neuen Studiengang zu konzipieren, der sich durch sein breites Angebot von klassischen geisteswissenschaftlichen Studien unterscheidet. Die Zuerkennung einer Förderung (für die Entwicklung des Schwerpunktes) seitens der deutschen VW-Stiftung, die im Allgemeinen Universitäten und Hochschulen innerhalb Deutschlands fördert, bestätigt die Relevanz eines solchen Konzeptes über nationale Grenzen hinweg und bestärkt die Grundhaltung und die Schwerpunkte der Olmützer Germanistik:

Die Wissenschafts-Grenzen verlaufen nicht identisch mit den politischen und Staatsgrenzen, außerdem verstand sich die Olmützer Germanistik mit ihren wissenschaftlichen Schwerpunkten und dem Streben, die Lehre sowohl in deren inhaltlichen und qualitativen Kriterien als auch in der organisatorischen Struktur an deutschen Vorbildern zu messen, immer als ,Inlandsgermanistik', hiermit bewusst an die Tradition der böhmischen Germanistik vor 1945 anknüpfend. ${ }^{2}$

\section{Struktur des Studienplans}

Der Studiengang „Deutsch als Sprache der Geisteswissenschaften“ ist, wie schon erwähnt, als Schwerpunkt oder Vertiefung Teil des Germanistik-Studiums. Zusätzlich zu den germanistischen Lehrveranstaltungen sind, je nach Studienjahr, weitere Lehrveranstaltungen zu absolvieren (bzw. wurde der Studienplan insgesamt

1 Über die Struktur des Studienschwerpunktes wurde am Lehrstuhl eine tschechischsprachige Broschüre erstellt, die sich vor allem an potentielle Studierende richtet. Die hier und im Folgenden zitierten Auszüge stammen dagegen aus der Beschreibung des Konzeptes von Ingeborg Fiala-Fürst, die in deutscher Sprache auf der Homepage der Germanistik an der Palacký-Universität nachzulesen ist. Die genaue Adresse lautet: http://www.germanistika.cz/index.php?option=com_content\&view= article\&id=39 (Stand: 20. 06. 2011).

2 http://www.germanistika.cz/index.php?option=com_content\&view=article\&id=39 (Stand: 20. 06. 2011). 
adaptiert). ${ }^{1}$ Im ersten Jahr sind dies neben einschlägigen Sprachübungen, auf die ich noch näher eingehen werde, Lehrveranstaltungen zu sechs geisteswissenschaftlichen Disziplinen: Philosophie, Geschichte, Allgemeine Sprachwissenschaft, Religionswissenschaft (Schwerpunkt Judaistik), Soziologie und Mediävistik, jeweils drei in einem Semester. Am Ende des ersten Studienjahres bzw. am Beginn des nächsten sollen die Studierenden aus diesen sechs Fächern eines auswählen, das sie im weiteren Studium vertiefen.

Die Lehrveranstaltungen zu den einzelnen geisteswissenschaftlichen Disziplinen können natürlich nicht mit den jeweils vollwertigen Studiengängen gleichgesetzt werden oder diese ersetzen, was auch keinesfalls das Ziel des Studienschwerpunktes ist. Vielmehr sollen die Studierenden neben dem regulären Germanistikstudium (bzw. damit verbunden) Grundwissen aus den einzelnen Disziplinen erwerben, für methodologische Fragen und Spezifika sensibilisiert werden (etwa für den Umgang mit Quellen in der Geschichtswissenschaft) und vor allem einen stark interdisziplinären Ansatz entwickeln. ${ }^{2}$

An dieser Stelle muss angemerkt werden, dass mit dieser sehr umfassenden Wissensvermittlung nicht zuletzt eine Lücke gefüllt werden soll, die in den letzten Jahren und Jahrzehnten immer größer zu werden scheint:

Ohne die Gründe dafür genau orten zu wollen und können, bemerken wir in den letzten, etwa zehn Jahren bei den Studierenden der ersten Semester eine erschreckende Abnahme des Basis-Wissens, dessen was man früher gemeinhin Bildung/Allgemeinbildung nannte. Da wir - trotz anderweitiger modischer Beteuerungen über die heutige Vorrangigkeit anderer Werte und Fertigkeiten im ,Bildungs- und Wissenschaftsbetrieb', der Wissensmobilität etwa, der Teamfähigkeit, der Eloquenz, der Wendigkeit im Umgang mit elektronischen Netzen usw. - nach wie vor glauben, dass Allgemeinbildung wichtig ist für einen Philologen, [...] wollen wir die Lücken, die die Schulbildung in allen Sparten hinterließ, innerhalb des Germanistik-Studiums zu schließen versuchen. ${ }^{3}$

Nebenbei bemerkt: Dieser Befund (schwindendes Allgemeinwissen) trifft durchaus nicht nur auf die Tschechische Republik zu, sondern auch auf Länder wie z. B.

1 Dies gilt für Studierende, die dieses Studium als Einzelfach belegen. Wer Germanistik als Zweitfach (neben z. B. Anglistik, Bohemistik u. a.) inskribiert, hat die Fächer des Schwerpunktes nicht zu belegen.

2 Genau dieser breite Ansatz, der sowohl fachliches Grundwissen in unterschiedlichen Disziplinen als auch fächerübergreifende Kompetenzen vermittelt, ist im Grunde viel näher an der Ursprungsidee der neuen Aufteilung eines Studienverlaufes (in ein Bakkalaureats- und ein Masterstudium), als es so manche halbherzigen Umstrukturierungen in Deutschland und Österreich sind: Eine breite Grundbildung im Bakkalaureat, der eine Spezialisierung (erst) im Master folgt.

3 http://www.germanistika.cz/index.php?option=com_content\&view=article\&id=39 (Stand: 20. 06. 2011). 
Österreich, wie es die immer gleichen Bildungsdebatten in Folge von verschiedenen Leistungstests (am prominentesten: PISA) in schöner Regelmäßigkeit aufzeigen. ${ }^{1}$

Aus sprachdidaktischer Perspektive (und diese soll hier im Vordergrund stehen) ist die Tatsache hervorzuheben, dass die unterschiedlichen wissenschaftlichen Disziplinen über ein jeweils mehr oder weniger spezifisches Fachvokabular verfügen, das es zu vermitteln gilt, um den Studierenden eine auch sprachlich fundierte Auseinandersetzung mit Themen und Fragestellungen der jeweiligen wissenschaftlichen Arbeitsbereiche zu ermöglichen. Ein zentrales Anliegen dabei ist aber ein fächerübergreifendes Grundvokabular wissenschaftlichen Schreibens und Sprechens. Dem sind eigene Sprachübungen gewidmet, die in den ersten beiden Semestern jeweils vier Wochenstunden umfassen. Zum einen wird dabei mit konkreten Texten gearbeitet, vergleichbar mit Textarbeit im konventionellen DaFUnterricht. ${ }^{2}$ Zum anderen aber haben Karsten Rinas, Birgit Gunsenheimer und Veronika Opletalová eigens für den Studienschwerpunkt ein Lehr- und Übungsbuch erstellt, das an dieser Stelle eingehender vorgestellt werden soll. Derzeit wird eine Probefassung im Unterricht getestet, die endgültige Fassung ist noch ausständig, wird aber nach bisherigen Ergebnissen mit nur unwesentlichen Änderungen erscheinen (alle Zitate in diesem Text beziehen sich auf die Probefassung). Diese Ausgabe wird an der Palacký-Universität erscheinen und auch für andere Germanistikinstitute bzw. Organisationen und Zentren der Sprachvermittlung verfügbar sein.

\section{Das Übungsbuch zur deutschen Wissenschaftssprache}

Das Übungsbuch zur deutschen Wissenschaftssprache geht über schon bestehende ähnlich betitelte Werke hinaus ${ }^{3}$ bzw. verbindet diesen Bereich mit einem anderen: der Produktion wissenschaftlicher Texte oder, wie es die Autoren in der Einleitung formulieren: „Dieses Übungsbuch soll Kenntnisse vermitteln, die sowohl für die Rezeption als auch für das Verfassen deutschsprachiger wissenschaftlicher Texte benötigt werden“ (Rinas, Gunsenheimer und Opletalová 2010: 5). ${ }^{4}$

1 Ich möchte beispielhaft auf ein Buch des österreichischen Philosophen Konrad Paul Liessmann verweisen, der auf einer höheren Ebene und mit Blick auf die universitäre Bildung die strukturellen Gründe einer veränderten Bildungs- und damit auch Wissenschaftslandschaft thematisiert: „Theorie der Unbildung - Die Irrtümer der Wissensgesellschaft" (Liessmann: 2006), das unter dem Titel „Teorie nevzdělanosti - Omyly společnosti vědění" im Jahr 2008 auch auf Tschechisch erschienen ist.

2 Allerdingskonzentriertsichauchdiese, Textarbeit' aufwissenschaftlichebzw.wissenschaftsjournalistische Texte. Berücksichtigt werden dabei möglichst viele der oben angegebenen Disziplinen.

3 Die Autoren verweisen in der Einleitung auf die doch recht dürftige Anzahl schon bestehender Bücher, die sich diesem Thema widmen und heben in einigen Fällen die teils ziemlich gravierenden Mängel hervor. Etwa bei dem „wohl am weitesten verbreitete[n] Werk dieser Art, d[er] Einführung in die deutsche Sprache der Wissenschaften": „De facto ist SCHADEs [2002] Einführung nur eine gewöhnliche deutsche Übungsgrammatik für Fortgeschrittene, die die Spezifika der Wissenschaftssprache kaum thematisiert." (Rinas, Gunsenheimer und Opletalová 2010: 5).

4 Teil 5 des Buches, den Birgit Gunsenheimer erarbeitet hat, widmet sich sehr ausführlich der 
Die ersten beiden Teile des Buches sind als Hinführung zur Thematik zu verstehen. Sie dienen also einerseits der Sensibilisierung für das Thema ,Fachspezifische Terminologie ${ }^{11}$ und besprechen andererseits die Strukturierung wissenschaftlicher Texte. Die Vorgehensweise bei Letzerem ist zweigeteilt: Einer theoretischen Definition (oder mehreren unterschiedlichen Definitionen) von Strukturelementen eines Textes folgen konkrete Beispiele; beides ist mit Arbeitsaufgaben bzw. Fragen verbunden. So finden sich etwa zum Vorwort zunächst drei Definitionen von Niederhauser (2006), Rückriem, Stary und Franck (2006) sowie Poenicke (1988), anschließend zwei Beispiele für Vorworte, eines aus Anke Sauters (2000) Eduard Engel. Literaturhistoriker, Stillehrer, Sprachreiniger und ein weiteres aus Helmut Seiferts (1977) Stil heute. Eine Einführung in die Stilistik.

Das Thema ,wissenschaftliche Sprache erschöpft sich aber natürlich nicht nur in der Behandlung von Fachtermini - das Übungsbuch umfasst zum Beispiel auch Fragen der Stilistik. Hier wird zum einen wiederum die Problematik von Internationalismen bzw. Fremdwörtern erörtert, aber auch ein ,typisch deutscher Un-Stil', nämlich der sogenannte Nominalstil, thematisiert. Auch dies ist ein Bereich, der sehr wohl auch innerhalb der deutschsprachigen Länder (also für Muttersprachler) von Interesse sein kann oder jedenfalls diskutiert werden sollte (was in einschlägigen Handbüchern zum wissenschaftlichen Schreiben, die sich an deutschsprachige Studierende richten, durchaus gemacht wird). Ein weiterer Aspekt des Lehrwerkes ist die Unterscheidung der fachwissenschaftlichen von der populärwissenschaftlichen (bzw. wissenschaftsjournalistischen) Sprache und ihrer jeweils unterschiedlichen Funktionen.

Angesprochen werden außerdem „Elemente der ,wissenschaftlichen Alltagssprache “" und ihre Verwendung. Was ist unter diesem Begriff zu verstehen? Es handelt sich dabei um Phrasen und Floskeln, die in schriftlichen, vor allem aber in mündlichen wissenschaftlichen Beiträgen häufig verwendet werden - bei Konferenzen, Vorträgen, Diskussionen; Muttersprachler sind sich dessen oft gar nicht bewusst. Dazu gehören zum Beispiel Phrasen, die der Textgliederung dienen:

- Einleitende Phrasen: Das Thema meines Vortrags ist ... / Einleitend möchte ich bemerken, dass ... etc.

- Wortverbindungen, mit denen zusätzliche Gesichtspunkte eingeführt oder betont werden: Des Weiteren möchte ich betonen ... I Es braucht wohl nicht besonders hervorgehoben werden ... / Wir sollten nicht unbeachtet lassen ... etc.

„Literaturrecherche als Teil des wissenschaftlichen Arbeitsprozesses" (Rinas, Gunsenheimer und Opletalová 2010: 155).

1 Wobei viel Raum den Internationalismen gewidmet wird, die in der wissenschaftlichen Sprache eine noch größere Rolle spielen als in der Standardsprache; einerseits können sie durchaus zu einem besseren oder schnelleren Textverständnis dienen, zum anderen sind sie aber nicht selten auch Quelle von Missverständnissen. 
- Ein neues Thema oder Teilthema einführende Worte: In diesem Zusammenhang sollte auch auf die Frage eingegangen werden, ob ... I Lassen Sie mich in aller Kürze noch auf die Frage eingehen ... etc.

- Abschließende Worte: Zum Abschluss möchte ich noch einmal betonen, dass ... I Aus dem hier Gesagten folgt, dass ... etc.

Diese vier Beispiele dienen nur der Illustrierung, im Lehrbuch selber finden sich viele weitere Beispiele. Besonders praxisorientiert sind dabei Phrasen und Wortverbindungen, wie sie zu Diskussionen benötigt werden. Die Bitte um Stellungnahme, die mögliche Reaktion auf Nachfragen und Einwände, das Formulieren von Bewertungen, die thematische Lenkung einer Diskussion, das Unterbrechen des Diskussionspartners und das Abwehren von Unterbrechungen - für alle diese möglichen Diskussionssituationen finden sich zahlreiche Ausdrucksmittel. Natürlich soll dies nicht ein starres Wiederholen stereotyper Phrasen bewirken, es ist vielmehr als Reservoir gedacht, an welchem sich Deutsch-Lernende orientieren können und damit auch eine Reihe von geeigneten Wortverbindungen zur Verfügung haben, mit denen sie sich fließend auch an spontanen Diskussionssituationen beteiligen können.

Gerade in diesem Zusammenhang wird auch am stärksten deutlich, dass das Lehrbuch für tschechische Deutsch-Lernende gestaltet wurde. Jeder der angeführten Formulierungen ist zusätzlich eine tschechische Übersetzung bzw. ein tschechisches Äquivalent beigestellt. Im Vergleich werden die ,Fallen' deutlich, die tschechischen Deutsch-Lernenden in solchen Fällen drohen. Wie Karsten Rinas (2003: VII) es formuliert:

Wohl jeder DaF-Lehrer, der schon einmal eine Gruppe von Lernern mit gleicher Ausgangssprache unterrichtet hat, wird die Erfahrung gemacht haben, dass es eine Reihe von ,Standard-Fehlern' gibt, die von den Lernern immer wieder gemacht werden. Bei vielen dieser Fehler handelt es sich um Interferenzfehler, also um Fehler, die durch Besonderheiten der Muttersprache der Lerner evoziert werden.

Einer dieser Interferenzfehler, den ich schon fast ,klassisch ' nennen möchte, weil er im Unterricht unweigerlich vorkommt (und zwar meist früher als später), ist die Frage: Darf (oder: Kann) ich eine Frage haben? (Tschechisch: Müžu mit otázku?).

Solchen Interferenzfehlern, die in unterschiedlichen grammatischen und/oder semantischen Strukturen des Deutschen und des Tschechischen begründet sind, wird im vierten Teil des Buches ebenfalls viel Platz eingeräumt. Dafür konnte unter anderem auf das reichhaltige Material zurückgegriffen werden, das Karsten Rinas im Zuge seiner Forschungen über Interferenzfehler tschechischer Deutsch- 
Lernenden gesammelt hat. ${ }^{1}$ Für das Übungsbuch hat er neben den schon erwähnten Phrasen und Wortverbindungen noch einige besonders Fehler-anfällige Wörter und Wortverbindungen des Tschechischen ausgewählt; er erläutert dabei einerseits die Problematik und führt andererseits mögliche deutsche Äquivalente an. Beispiele dafür wären etwa zminit se o čem, das dem deutschen etwas erwähnen entspricht. Das Verb erwähnen ist aber (im Deutschen) eben nicht reflexiv, was leicht zu Fehlern führen kann. Gründe für unglückliche und fehlerhafte Übertragungen des tschechischen Wortes ř rě̌itsind dagegen in der Semantik zu finden: Dieses Wort kann im Tschechischen in unzähligen Kontexten verwendet werden, die im Deutschen nicht mit dem eigentlichen Wortäquivalent lösen übersetzt werden können. Solche Interferenz-Aspekte können im Unterricht einerseits durch Modelldiskussionen thematisiert und didaktisiert werden, andererseits durch Übersetzungsübungen einschlägiger Textbeispiele.

Der Vollständigkeit halber soll noch erwähnt werden, dass das Buch mit komplexeren Textübungen schließt, deren Struktur auf die Abschlussklausuren vorbereiten soll, die am Institut für Germanistik der Palacký-Universität am Ende des ersten und zweiten Studienjahres durchgeführt werden. In diesem Sinne sind auch die vier Musterklausuren am Ende des Buches zu verstehen, die durch das Bereitstellen von Lösungsvorschlägen ein sinnvolles Überprüfen der eigenen Fähigkeiten (der Studierenden) ermöglicht.

Abschließend möchte ich noch einmal darauf hinweisen, dass diese hier (schwerpunktorientiert) präsentierten Aspekte einer intensiven und sehr praxisnahen Vermittlung von fächerübergreifenden sprachlichen Elementen der wissenschaftlichen Kommunikation immanenter Teil des Gesamtkonzeptes Deutsch als Sprache der Geisteswissenschaften sind: Die Studierenden sollen nicht nur fachspezifisches Vokabular, sondern darüber hinaus (also fachübergreifend) Kompetenzen des wissenschaftlichen Arbeitens, vor allem aber eines adäquaten sprachlichen Ausdruckes erwerben. Dies sollte nicht nur bei Aufgaben im universitären bzw. studentischen Alltag (Referate, Seminar- und Abschlussarbeiten) zu besseren Ergebnissen führen, sondern die Studenten auch befähigen, ihre wissenschaftlichen Arbeiten auch außerhalb der eigenen Universität (bzw. nach vollendetem Studium) zu präsentieren und zu diskutieren.

\section{Literaturverzeichnis}

Liessmann, Konrad Paul (2006): Theorie der Unbildung - Die Irrtümer der Wissensgesellschaft. Wien, Zsolnay.

1 Erwähnt sei an dieser Stelle die Publikation Interferenzfehler deutschsprechender Tschechen, deren dritter Teil sich "Sprachliche[n] Auffälligkeiten in wissenschaftlichen Fachtexten" widmet (Rinas 2003). 
Liessmann, Konrad Paul (2008): Teorie nevzdělanosti - Omyly společnosti védění. Übers. aus dem Deutschen von Jana Zoubková. Praha, Nakladatelství Academia.

Niederhauser, Jürg (2006): Die schriftliche Arbeit-kurz gefasst. Eine Anleitung zum Schreiben von Arbeiten in Schule und Studium. Mannheim, Leipzig [u. a.], Dudenverlag, 4. Aufl.

Payrleitner, Alfred (1990/2003): Österreicher und Tschechen. Alter Streit und neue Hoffnung. Wien [u. a.], Böhlau, 2. Aufl.

Poenicke, Klaus (1988): Wie verfaßst man wissenschaftliche Arbeiten? Mannheim, Wien, Zürich, Dudenverlag, 2. Aufl.

Rinas, Karsten / Gunsenheimer, Birgit / Oletalová, Veronika (2010): Übungsbuch zur deutschen Wissenschaftssprache. Olomouc (Probefassung).

Rinas, Karsten (2003): Interferenzfehler deutschsprechender Tschechen. 3. Teil: Sprachliche Auffälligkeiten in wissenschaftlichen Fachtexten. In: Brünner Beiträge zur Germanistik und Nordistik, R 8, 2003. S. 31-74.

Rinas, Karsten (2001): Wenn Sie sagen: "Ich brauche mehr Deutsch üben“-dann haben Sie Recht! Programmierte Übung zum Verlernen typisch tschechischer Deutsch-Fehler. Opava, Slezská univerzita v Opavě. Ústav cizích jazyků.

Rückriem, Georg / Stary, Joachim / Franck, Norbert (2006): Die Technik wissenschaftlichen Arbeitens. Paderbon, München [u. a.], Schöningh.

Sauter, Anke (2000): Eduard Engel. Literaturhistoriker, Stillehrer, Sprachreiniger. Bamberg, Collibri-Verlag.

Schade, Günter (2002): Einführung in die deutsche Sprache der Wissenschaften. Berlin, Erich Schmidt, 12. Aufl.

Seifert, Helmut (1977): Stil heute. Eine Einführung in die Stilistik. München, C.H. Beck.

\section{Quellen aus dem Internet}

http://www.germanistika.cz/index.php?option=com_content\&view=article\&id=39 (Stand: 20. 06. 2011) 